\title{
Contents, Vol. 1, 1899
}

$-2-$

Silex, P., Rundzellensarkom in einem phthisischen Bulbusbei einem 7jährigen Kinde nebst therapeutischen Be-merkungen

Wilbrand, II., Ueber die diagnostische Bedeutung desPrismenversuchs zwischen der basalen und der supra-nucleären homonymen Hemianopsie

Casuistisehe Mitteilung·en.

Ellinger, A., Myxosarcom des Sehnerven. Operiert nacliKrönlein rait Erlialtung des Bulbus

- Ein Fall von Empyem des Sinus frontalis mit operative!-Beseitigung der liinteren

Knoclienwand wegen Caries

Kulmt, Hermann, Erfolgreiehe Bekämpfung e-iner Corneal-eiterung (Pneumokokken-Infection) post extractionem

Sclioute, G $\gamma$. J., Ein Fall von Cornealruptur mit Iris-perforation ohne $\Lambda \tau$ erletzung der Linse Sammelreferate.

Ueber die Diagnostik und Extraction von Eisensplittern.

Von Dr. Ed. Asmus in Düsseldorf

Sammelreferat der bacteriologischen Arbeiten der Jalire 1897

und 1898. Von Priv.-Doc. Dr. L. Bach und Dr.

R. O. Neumann in Würzburg

Semestralbericht über die amerikanische Litteratur. 1. Januar

bis 1. Juli 1898. Von R. Denig in New-York 395. 508 u. Bericht über die russisclie Litteratur des Jahres 1898, I. Teil

IL “

Von Prof. Dr. von Ewetzki in Moskau.

Bericht über die ungarische ophthalmologische Litteratur

$3 / 8$

des Jahres 1898. Von Doc. Dr. Em. v. Grrósz inBudapest

Ueber die Entwickelung und den heutigen Stand unsererKenntnisse von der pathologischen

Anatomie undAetioloa;ie des Trachoma. Von Dr. P. Junius inKönigsberg

Bericht über die polnische ophthalmologische Litteratur vom1. 7. 1898 bis 1. 1. 1899. Erstattet

von Dr. Kamockiin Warschau

Die subconjunctivalen Injectionen. Von Prof. Dr. CarlMellinger in Basel

Einige neuere Arbeiten zur Theorie der Sebschärfe. VonDr. F. Schenck in Würzburg

Die operative Behandlung der hochgradigen Myopie. VonDr. K. Schrader in Gera

Die Beziehungen der Augenstörungen zu den Nervenkrank-heiten. Bericht für das Jahr 1897.

Von Prof. Dr.P. Silex in Berlin

$-3-$

Bericht über die scandinavische ophthalmologische Litteraturdes II. Semesters 1898. Von Prof.

Dl . Widmark inStockholm . 302

Sitzung $\cdot$ sbepiehte. 
Berliner ophthalmologische Oxesellschaft:

Sitzung vom 20. April 1899532

Berliner physiologische Gesellschaft:

Sitzung vom 24. Februar 1899

Berliner Gesellschaft für Psychiatrie und Nerven-krankheiten:

Sitzung vom 13. März 1899 ..... •.. 408

„8. Mai 1899 617

Chicago Ophthalmological and Otological Society:

Sitzung vom 8. November $1898 \quad 114$

„' „, 13. Dezember 1898

„10. Januar 1899 . . . . . . 406

„11. März $1899 \quad 531$

Denver and Arapahoe Medical Society:

Sitzung vom 11. April 1899 ..616

Bericht über die ophthalmologische Section der Natur-forscherltersammlung in Düsseldorf vom

19. bis

24. September $1898 \quad 103$

Ophthalmological Society of the United Kingdom:

Sitzung vom 26. Januar 1899306

The New-York Academy of Medecine. Section of Ophthalmology:

Sitzung vom 16. Januar 1899 .

New-York county Medical Association:

Sitzung vom 19. Dezember 1898215

Société d'Ophthalmologie de Paris:

Sitzung vom 7. März 1899 • .528

Philadelphia County Medical Society:

Sitzung vom 12. April 1899616

College of Physicians of Philadelphia. Section of Ophthalmology:

Sitzung vom 15. November $1898 \quad 114$

„20. Dezember 1898305

„17. Januar $1899 \quad 530$

San Francisco Society of Eye, Ear, Nose and Throat Physicians:

Sitzung vom 19. Januar 1899531

Washington (S. C.) Society of Ophthal rnologists and Otologists:

Sitzung vom 29. April 1899616

-4 -

Therapeutische Umsehau.

Therapeutische Umsehau . . . 112, 216, 307, 409, 533, 617

Unfall- und Sachverständ $1 / 8$ enkunde.

Unfall- und Sachverständigenkunde 414, 536, 621

Buehanzeigren.

Haab, R., Atlas der äusseren Erkrankung des Auges, nebst

Grrundriss der Pathologie und Therapie ..... 221

Praun, E., Die Yerletzungen des Auges 625

Seligmann,S., DiemikroskopischenUntersuchungsniethoden

des Auges 118 
Wilbrand, H. und A. Saenger, Die Neurologie des

Auges 115

Neerolog $\cdot \mathrm{e}$.

Alfred Graeíe 627

Julius Samelsohn 419

Tag·esnachpichten und Pérsonalien.

Tagesnaclu · ichten und Notizen . 119, 223, 309, 420, 539, 629

Litteratup-Vepzeiehniss.

Litteratur-Verzeichnis .... 119, 223, 310, 420, 540, 630

Zeitschrift für Augenheilkunde.

Unter ständiger Mitarbeit von

Dr. Denig-New-York, Prof. v. Ewetzki-Moskau,

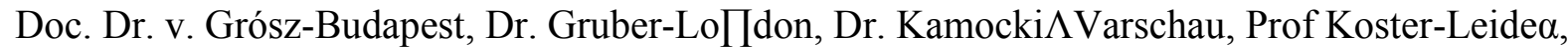

Dr. Pergens-Brüssel, Prof. Widmark-Stockholm u. A.,

herausgegeben von

Doe. Dr. Bach- Würzburg, Prof. Czermak-Prag, Prof. Dimmer-Innsbruck,

Prof. 0. Haab-Zürich, Prof. Kuhnt-Königsberg, Prof. Mellinger-Basel,

Prof. v. Michel- Würzburg, Prof. Hermann Pagenstecher-Wiesbaden, Prof. Peters-Bonn,

Prof. Raehlmann-Doipat. Prof. Schmidt-Rimpler-Güttingen, Prof. Silex-Borlin,

Prof. Uhthoff-Breslau, Prof. VOSSiUS- Giessen, Dr. Wilbrand-Hamburg,

redigiert von

Prof. Dr. H. Kuhnt u ud Prof. Dr. J. von Michel

in Königsberg i. Pr. in Würzburg.

Band I.

Berlin 1899.

VERLAG VON S. KARGER

KARLSTRASSE $\tau 5$.

Mit vielen Abbildungen hn Text un $<14$ Tafeln.

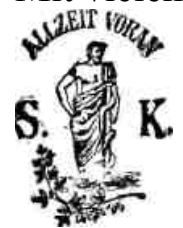

Inhalts-Verzeichnis.

Originalarbeiten.

Axenfeld, Tli., Ueber Luxation, Zerstörung und Heraus-reissung des Augapfels als

Selbstverstümmelung beiGeisteskranken. (Hierzu ïafel II) 128

Bach, Ludwig, Zusammenfassende Darstellung und kritischeBetrachtung der Erkrankungen der Vierhügelgegendund der Zirbeldrüse mit specieller Berücksichtigungder ocularen Symptome ... 315,455

- $\quad$ Bemerkungen zur Pathogenese der sympathischen Oph-

thalmie . 353

- $\quad$ Sind die nach Infectionen und Intoxicationen auftretenden

Augenmuskellähmungen als periphere oder als centrale

Lähmungen aufzufassen? 548

Back, S., Ueber leukämisclie Augenveränderungen. (Hierzu

Tafel III) 234 
Burri, Roman, Wirkung subconjunctivaler Kochsalzinjectionen bei Chorioiditis in macula. (Hierzu Tafel I) . 21 Diez, W., Beitrag zur Aetiologie der Keratitis parenchymatosa 435, 551

Erb, A., Ein Fall $\tau$ on Spontanausstossung eines Zündhütchenstückes aus dem Auge 5 Jahre nach der Verletzung 449 Пallauer, Otto, Ueber das Rotwerden des Eserins . . . 364 Hauenschild, Wilh., Ueber Antisepsis und Asepsis bei

Bulbusoperationen, nebst kurzen Bemerkungen zur

Nachbehandlung Staaroperierter 227

Hess, C, Ueber fötale Rupturen der hinteren Linsenkapsel

und über Lenticonus posterior. (Hierzu Tafel TV) . 427 Junius, P., Ueber das Vorkommen der acuten Pneumo-

kokken-Cônjunctivitis43

Koster, W., Ein neuer Sperr-Elevateur. (Mit 6 Ab-

bildungen) 245

- $\quad$ Eine neue Methode der Ptosisoperation. (Mit 6 Ab-

bildungen) $\quad 543$

Kuhnt, Hermann, Ueber den Heihvert der mechanischenMethoden in der Therapie der

Conjunctivitis granulosa.(Mit 4 Abbildungen)

1

Zur Kenntnis der acuten Methylalkohol-Intoxication . . 38

Ueber Nachstaaroperationen. (Mit 10 Abbildungen) 151, 260

E. Eine Modification der Anwendungsweise des Expressors

bei der Conjunctivitis granulosa. (Mit 3 Abbildungen) 359 